\title{
Trabajos libres
}

\section{TRANSICIÓN OCUPACIONAL,}

\section{EL ADULTO MAYOR A LA UNIVERSIDAD}

Angélica María Monsalve Robayo

Terapeuta Ocupacional. Master en Gerontología

Profesora Programa de Terapia Ocupacional

Universidad del Rosario

\section{Palabras claves}

Jubilación, participación social, ocio productivo, ámbitos educativos, adulto mayor.

El proyecto denominado Transición ocupacional, el adulto mayor a la universidad se formula con el ánimo de proponer nuevas alternativas de desarrollo de esta población profundizando sobre los cambios que se evidencian tras el proceso de jubilación y lo que esto implica para los adultos mayores en términos de hábitos, toma de decisiones sobre actividades familiares, posiciones sociales frente a problemáticas específicas, accesibilidad, manejo productivo del tiempo, entre otros.

Para esto se retoman antecedentes históricos sobre la implementación de programas educativos a nivel nacional e internacional, profundizando acerca de iniciativas tales como la Universidad de la Tercera Edad en Toulouse, Francia; la Universidad de la Experiencia en Salamanca-España, Educación permanente en la Universidad de Santiago de Chile, entre otras.
El objetivo de este proyecto es brindar una alternativa ocupacional que permita no solo la adquisición de conocimientos sino alcanzar un desarrollo personal a través de la socialización de experiencias de vida y la participación activa entre generaciones, contribuyendo al posicionamiento y reconocimiento del adulto mayor en la sociedad.

En el proyecto han participado asistentes de investigación de los programas de Terapia Ocupacional y Fisioterapia de la Universidad del Rosario, quienes han aportado al desarrollo de las tres fases del proyecto (exploratoria, analítica y operativa), las cuales contemplan la revisión conceptual de las categorías de ocio y tiempo libre, educación como medio de participación en el adulto mayor, aprendizaje, jubilación y vinculación socio-cultural; el conocimiento sobre las expectativas de la 
población potencial y las experiencias previas sobre este tipo de iniciativas para la elaboración y puesta en marcha del programa a desarrollar.

Los componentes del proyecto incluyen una mirada sociocultural, de participación universitaria y de aprendizaje y actualización que fundamentan el desarrollo de estrategias de integración generacional y participación en procesos formativos respondiendo a necesidades y expectativas de este grupo poblacional.

Es por esto que se visualiza el aprendizaje en esta población como un proceso implícito a lo largo de la vida, entendiendo así que cada etapa del ciclo vital tiene características que facilitan el desarrollo del mismo y por ende la adquisición de diferentes conocimientos y de exploración de un entorno determinado.

Para el desarrollo del proyecto se contó con la participación de asociaciones de pensionados para conocer a través de una encuesta de opinión los intereses y la percepción de la población potencial hacia este tipo de programas educativos.

En este sentido el proyecto se circunscribe a una metodología que orienta el cambio social a través de la participación y la acción que se observa implícita en los problemas reales y enfatiza en la resolución de un problema concreto en el aquí y en el ahora en una situación determinada.

Al analizar los resultados de esta encuesta de opinión se visualiza un inte- rés predominante hacía la participación en procesos de formación en áreas tales como la tecnología, los idiomas, las artes, los proyectos de microempresa, cuidado de la salud y cultura general.

En este sentido se diseña un programa que abarca contenidos y experiencias en los diferentes escenarios de participación que promueve la Universidad del Rosario y que busca a través de su proyecto educativo institucional y de las políticas de proyección social, prestar servicios integrados para la solución de los problemas, desde el marco de acción permisible en razón de las tareas y actividades de las diferentes facultades que constituyen la Universidad. Esto a su vez ayudará a obtener una orientación interdisciplinaria en la formación profesional y apoyará la formulación de nuevos programas interdisciplinarios, de educación continuada y de educación no formal ${ }^{1}$.

La propuesta de programa que aún se encuentra desarrollándose y estudiándose a razón de los tiempos, costos y metodologías, incluye espacios de participación en los tres componentes del proyecto incluyendo asignaturas tales como expresión corporal, primeros auxilios, musicoterapia, salud y medio ambiente, imagen profesional, memoria y sociedad, economía y sociedad, sociología de la educación, sociología de la empresa, mundo del

1 Universidad del Rosario. Proyecto Educativo Institucional. Bogotá, enero 2005. Pág.15. 
trabajo, tecnología en cartón, estrategia empresarial, introducción a la administración, seminario inicie su empresa, sistemas de información, introducción al mandarín, taller de pintura, tango, entre otras.
Finalmente se espera concluir este proyecto con un programa educativo de interés colectivo que genere impacto a nivel social y promueva el uso productivo del tiempo libre, las relaciones intergeneracionales y la participación activa de la población adulta mayor.

\section{Bibliografía}

(1) Álvaro, José Luis; Garrido. Alicia; Torregrosa. Psicología Social Aplicada. Ed. McGraw-Hill, 1999 España.

(2) Artículo en formato electrónico. El recreo humano. Available from: URL:http://www. redcreacion.org.

(3) Información en formato electrónico. Down 21, Concepto de ocio. Available from: http:/ /www.down21.org

(4) Información en formato electrónico. PUCMM, programa de educación superior para adultos mayores. Available from: http:/ /www. pucmmsti.edu.do

(5) Gascón Pilar et al. Idiomas una alternativa de ocio en verano. Revista sesenta y mas: 6-11.

(6) Artículo en formato electrónico. Aprendiendo a envejecer. Available from: http://www. gerontologia.uchile.cl

(7) Álvaro, José Luis; Garrido, Alicia; Torregrosa. Psicología social aplicada. Ocio. Ed McGraw-Hill, 1999 España.

(8) Artículo en formato electrónico. Tiempo libre como calidad de vida en el adulto mayor. Avilable from: http://www.members. fortunecity.es

(9) Sánchez, Ocaña Ramón. Reflexiones optimistas de la vejez. Revista sesenta y mas: 3-8.

(10) Artículo en formato electrónico. Educación permanente no formal para adultos mayores. Available from: http://www.geragogia. net.

(11) Artículo en formato electrónico. Vejez, jubilación y el mito social. Available from: http:/ /www.psicomundo.com

(12) Artículo en formato electrónico. Tiempo libre como calidad de vida en el adulto mayor. Avilable from: http://www.members. fortunecity.es

(13) Artículo en formato electrónico. La necesidad del ocio. Available from: http://www. latinsalud.com

(14) Urrea, Maria Victoria. Evolución histórica y cultural de la ocupación en la tercera edad. Revista ocupación humana: 46-55.

(15) Dunleavey Taira, Ellen. Planificación de la jubilación. Ed. Panamericana

(16) Romieux Olarte, Michel. La Educación para el Adulto Mayor y su Relación con la sociedad. Available from: http:// www.rehue. csociales.uchile.cl

(17) Revista mayores al día. De profesión... animador socio-cultural. Revista mayores al día: 6-8.

(18) Información en formato electrónico. El aprendizaje a lo largo de la vida: la universidad de la experiencia. Available from: http:// www.lacerca.com 
(19) VILLA, Juan Manuel. Los mayores tenemos tiempo y libertad para disfrutarlo. Revista sesenta y mas: 24-27.

(20) Subirats, Joan. La vejez como oportunidad. La vejez como oportunidad: 17-61.

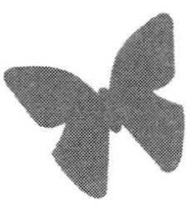

(21) Artículo en formato electrónico. Seres integrados. Available from: http://www. latinsalud.com

(22) Artículo en formato electrónico. El ocio un negocio, su misma negación. Available from: http:// www.chasque.net

(23) Vanguas Lezaun, Javier; Sancho Castello, Maria Teresa; Leturia Arrazola, Francisco Javier. Aspectos sicológicos del envejecimiento. Terapia ocupacional en geriatría: 65-89.

(24) Bazo, Maria Teresa; García Sanz, Benjamín; Maiztegui Oñate, Concepción; Martínez Paricio, Jesús. Envejecimiento y sociedad una perspectiva internacional. Ed. Panamericana: 86-95.

(25) Bazo, Maria Teresa; García Sanz, Benjamín; Maiztegui Oñate, Concepción; Martínez Paricio, Jesús. El medio ambiente habitual considerado para la tercera edad. Revista Ocupación Humana: 37-47.

(26) Molinero Aguilera, Silvia. Fragilidad en la esfera social. Masson S.A. 1996: 21-25.

(27) Márquez, Jaime. El envejecimiento como acontecimiento biológico en el ciclo vital. Revista de la asociación colombiana de geriatría y gerontología: 7-10.

(28) Artículo en formato electrónico. El adulto mayor como recurso. Available from: www. cps.unt.edu

(29) Información impresa. Perilla Santamaría, Sonia. A estudiar con canas y ganas. Diario: el Tiempo Nov 2002: 2-9.

(30) Información en formato electrónico. Universidad y adulto mayor. Available from: http:/ /www. educación.upa.cl 